\title{
Knowledge, practice and adverse reactions amongst Hair dye users - A cross sectional study
}

\author{
Research Article
}

\section{Mrudula Vinayak Joshi1 ${ }^{*}$, Joshi Vinayak Nilkanth², Shital Rahul Rasane ${ }^{3}$}

\author{
1. Professor \& H.O.D, Ayurveda Samhita Siddhanta, 2. Professor \& H.O.D., Rognidan And Vikruti Vidnyan, \\ 3. Associate Professor, Ayurveda Samhita Siddhanta, \\ Dr.D.Y.Patil College of Ayurveda and Research Centre, Dr. D.Y.Patil Vidyapeeth (Deemed to be University), \\ Pimpri, Pune, Maharashtra, India.
}

\begin{abstract}
Introduction: Colour of hair is most conspicuous phenotype of human beings and plays an important role in one's overall physical beauty. Premature hair greying (PHG) has caused the increased use of various hair dyes in population though it has adverse effects. Aim- To assess knowledge, practice of hair dye users with purpose, frequency of use and adverse reactions if any on hair, scalp, skin. Methodology: A cross-sectional study was conducted on participants from PCMC region by interview method using a questionnaire (20 questions) specially prepared pertaining to use of hair dye and adverse reactions to it. Statistical Analysis-The demographic data is expressed as mean \pm standard deviation, quantitative data expressed as percentages. Results: A total 98subjects including $75.5 \%$ and $24.5 \%$ females and males respectively. The participants started using hair dye at an age of 25 $( \pm 3.03)$ years. Herbal and Synthetic dyes were used by $36.74 \%$ and $63.27 \%$ respectively. Frequency of use in a year was $4-5$ times in $58.16 \%$. Adverse reactions of dye on body of hair like dryness $46.94 \%$, loss of hair $30.62 \%$, change in texture and thinning were reported. $29.59 \%$ of subjects showed ill effects during dyeing of scalp while in $68.36 \%$ of subjects after the dyeing process was complete. Conclusion: Majority Population started hair dyeing at very early age using Chemical dyes even though they perceived herbal dyes were safe. They continued hair colouring despite adverse reactions, which indicates aesthetic importance of hair colour and need of research in the field of Ayurveda for developing safe, natural hair dye.
\end{abstract}

Key Words: Herbal hair dye, chemical hair dye, adverse effects of hair dye, hair care, PHG, Ayurveda.

\section{Introduction}

The beauty of skin and hair basically depends on individual's health, diet, habits, job routine, climatic conditions etc. Skin and hair disorders are the common ailments of all age groups. Colour of hair is most conspicuous phenotype of human beings of all. Nowadays people are very much conscious about their look. Greying of hair is natural process of human aging. Grey /white hair develops naturally or prematurely in the process of human aging as a result of progressive and eventual total loss of melanocytes in the hair follicle. Hair with no melanin pigments in cortex is completely white and with few pigments provides grey colourl. Due to ever increasing problem of premature greying $(\mathrm{PHG})$ the hair dyes are used periodically for beautiful and younger look. Despite that use of hair dye leads to variety of side effects, there has been increased number of hair dye users in PCMC

\section{* Corresponding Author:}

\section{Mrudula Vinayak Joshi}

Professor and HOD, Department of Ayurveda Samhita \& Siddhanta, Dr. D.Y.Patil Vidyapeeth (Deemed to be University), Dr. D.Y. Patil College of Ayurveda and Research Center. Pimpri, Pune. Maharashtra. India.

Email Id: mrudula.doctor@gmail.com region. Hence this study was aimed at finding knowledge, purpose and adverse effects of hair dye among population.

Herbal dyes e.g. madayantika leaves, bhringraj etc are semi permanent dyes, used traditionally and believed to be safe and nontoxic. Plants have been used traditionally for their hair colouring, growth promoting and anti-aging properties. It has been found in the local market survey that the most of the marketed herbal formulations in India, though claim to be natural, safe and effective may actually contain the harmful synthetic agent, paraphenylenediamine (PPD), at $20-25 \%$ concentrations; which is the main ingredient of commercial synthetic dyes. (1) PPD, a key ingredient of many synthetic hair dyes is known to trigger allergic skin rashes in many people. It also causes dermatitis around lips, reddening and swelling of scalp and face etc. (2).

Ayurved treatises which described beautification of the hair as kesha prasadhana is a part of daily regimen. (3)Ayurveda defines keshya property i.e. beneficial for hair in terms of Vardhana (promotion of hair growth) and Ranjana (dyeing of hair). Kesaranjan action of drugs is also important to maintain aesthetic value of hair in keshavikar, Palitya (premature greying) which is very common nowadays. (4) It is described in an Ayurvedic text that ideal hair should be soft, unctuous, having strong roots and should be black(5).To propagate the main motive of Ayurveda towards healthy, beautiful hair and to overcome 
Mrudula Vinayak Joshi et.al., Knowledge, practice and adverse reactions amongst Hair dye users - A cross sectional study

the problem of palitya / PHG /Canities; it is very much essential to conduct research in this direction. As a first step in this direction the present survey study was conducted to assess the knowledge, practice, and adverse reactions among hair dye users.

\section{Aim}

To assess the knowledge, practice and adverse reactions among hair dye users in PCMC region.

\section{Objectives}

To find incidence of use of hair dye with purpose, frequency of use and to observe any adverse effects on hair, scalp, skin and eyes.

\section{Materials and methods}

This cross sectional survey was conducted on staff, students and their relatives from Pimpri, Pune region; with a specially prepared and authenticated questionnaire composed of 20 questions. The protocol was approved by the Institutional Ethics Committee. It was conducted over a period of 3 months from July to September 2018. Subjects from either sex with the age between 20 to 60 years and those who were willing to give the information were screened. Total 163 subjects with grey hair were surveyed and the response of 98 dye users who completed the protocol was used for data analysis. Percentage method was adopted for statistical analysis of quantitative data.

The questionnaire included basic information regarding gender, age, educational status, occupation, the age at which they first started using hair dyes, knowledge of hair colouring products and use of particular type of hair dye, frequency of its use, their purpose of colouring their hair, clinical findings of grey hair and hair dyeing-related adverse effects. Some question items were designed so that the survey subjects could make multiple choices.

\section{Results}

A total of $98(60.13 \%)$ out of 163 ; volunteers responded to the questionnaire. There were $75.5 \%$ females and $24.5 \%$ males representing the population (Table 1).

Table 1: Demographic data

\begin{tabular}{|l|l|}
\hline Variables & Frequency (\%) $\quad$ (n=98) \\
\hline \multicolumn{1}{|c|}{ Females } & 75.5 \\
\hline \multicolumn{1}{|c|}{ Males } & 24.5 \\
\hline Mean age years (SD) & $28.04 \pm 4.01$ \\
\hline $\begin{array}{l}\text { Marital status } \\
\text { Single }\end{array}$ & 39.8 \\
\hline Married & 60.2 \\
\hline Educational status & 100 \\
\hline Literate & 18.37 \\
\hline Graduates & 81.63 \\
\hline Post graduates & \\
\hline Occupation & 64.29 \\
\hline Employed & 35.71 \\
\hline Unemployed/ Student
\end{tabular}

SD - Standard deviation
All the volunteers were literate rather highly educated; with $64.29 \%$ employed and $35.71 \%$ unemployed / students. Observation about marital status (59 married, 39unmarried) was noted. The knowledge regarding the types (natural, synthetic, permanent etc.) and safe/preference use of hair dyes was noted. (Table 2)

Table 2: Knowledge about various types of hair dyes and personal use by participants

Knowledge of Hair Dye- Knowledge of Use of hair safe/ unsafe

hair dye dye Frequency \% Frequency \%

\begin{tabular}{|l|c|c|}
\hline Hair colouring was unsafe & 90.82 & 100 \\
\hline $\begin{array}{l}\text { Believed that dyeing could } \\
\text { cause cancer }\end{array}$ & 54.08 & 54.08 \\
\hline $\begin{array}{l}\text { Natural/ } \\
\text { Herbal dyes were safe }\end{array}$ & 80.61 & 36.74 \\
\hline \begin{tabular}{l|l|} 
Synthetic dyes were safe \\
Semi permanent dyes \\
were safe
\end{tabular} & 37.76 & 63.27 \\
\hline $\begin{array}{l}\text { Temporary dyes were safe } \\
\text { Permanent dyes were safe }\end{array}$ & 38.76 & 51.02 \\
\hline
\end{tabular}

$90.82 \%$ perceived that hair dyeing itself was unsafe yet only $36.74 \%$ used herbal dye whereas $63.27 \%$ used synthetic dye.

Practice regarding use of hair dye- The mean age at which the participants started using hair dyes was around $28.04 \pm 4.01$ years among population. $76.53 \%$ used hair dye to look younger, as compared to cosmetic purpose $(56.12 \%)$ who felt it was fashionable. (Table3).

Table 3: Purpose of Dyeing hair

\begin{tabular}{|l|r|}
\hline \multicolumn{1}{|c|}{ Purpose } & Frequency\% \\
\hline 1.For Fashion & 56.12 \\
\hline 2.Graying of hair & 21.43 \\
\hline 3.Younger look & 76.53 \\
\hline 4.Not specified & 2.04 \\
\hline
\end{tabular}

Area coverage by the dye was noted to be global and partial in 55 and 21 participants respectively. Maximum number i.e.57 participants (58.16\%) used hair dye at a frequency more than 4-5 times a year whereas 23 were habituated for dyeing before one month. Remaining used to do it at an interval of $7-15$ days. (Table 4).

Table 4: Frequency of dyeing hair and area covered by hair dye Frequency of dyeing hair after Count of participants $(\mathbf{n}=98)$
1) 7 days
3
2) 15 days
3) 1 month
23
4 ) More than 1 month

57

15

Area covered by hair dye

1-Global 55

2-Partial 21

3- Root touch-up 18

$1 \& 2$ 4 
Only $23.47 \%$ preferred to dye their hair at external source as compared with $74.43 \%$ did it at home. Majority $(80 \%)$ of the participants followed the instructions like patch test of hair colouring while only $10 \%$ never performed a skin test prior to the usage.

Attitude regarding use of hair dyes- Among the participants, $75 \%$ felt the need for repeated dyeing because of cosmetic value and gray hair. Almost $81 \%$ of the volunteers were satisfied with the colour after dyeing.

Adverse reactions of hair dyes on skin and hair - No immediate or severe adverse reaction was found in any of the participants but itching, burning, dryness and discoloration of scalp skin was noted after 24 hours in 23 participants. (Table5)

Table 5: Adverse effects during dyeing at scalp, upon surrounding skin and eyes

\begin{tabular}{|l|c|c|c|}
\hline $\begin{array}{c}\text { Adverse } \\
\text { effects }\end{array}$ & $\begin{array}{c}\text { During dyeing } \\
\text { at scalp } \\
\text { Frequency\% }\end{array}$ & $\begin{array}{c}\text { Upon } \\
\text { surrounding skin } \\
\text { Frequency\% }\end{array}$ & $\begin{array}{c}\text { Upon eyes } \\
\text { Frequency\% }\end{array}$ \\
\hline 1-Itching & 17.35 & 2.04 & 2.04 \\
\hline 2-Burning & 12.25 & 2.04 & 12.25 \\
\hline 3-Redness & 8.16 & 7.14 & 11.23 \\
\hline 4-Rash & 2.04 & 4.08 & - \\
\hline 5-NO & 76.53 & 89 & 74.50 \\
\hline
\end{tabular}

Adverse reactions of hair dyes on hair - This was an important and for the first time noted observation in similar type of studies. A total of 67 volunteers had reported adverse reactions on body of hair. Dryness of hair was most noticed effect in $46.94 \%$. Hair loss, thinning and dandruff $(30.62 \%, 22.45 \%$ \& $17.35 \%$ respectively) were very common in participants who used synthetic dyes. Changes in hair texture and discoloration were the major and long lasting ill effects as per (Table 6).

\section{Table 6: Adverse effects after dyeing on hair}

\begin{tabular}{|l|l|c|}
\hline Sr.No. & Adverse Effect on hair & Frequency \% \\
\hline 1 & Discolouration & 15.31 \\
\hline 2 & Dryness & 46.94 \\
\hline 3 & Hair loss & 30.62 \\
\hline 4 & Dandruff & 17.35 \\
\hline 5 & Thinning & 22.45 \\
\hline 6 & Splitting & 9.19 \\
\hline 7 & Changed texture & 14.29 \\
\hline 8 & No side effect & 31.63 \\
\hline
\end{tabular}

Survey subjects were allowed to make multiple choices.

Adverse reactions of hair dyes on body-Rhinitis and headache were commonly noticed side effects on the body. $82 \%$ participants did not suffered from any symptom may be suitable type of dye and time of application are the reasons for it. (Table 7)

Table 7: Adverse effects on general body after dyeing hair

\begin{tabular}{|l|c|}
\hline General Side effect & Frequency $\%$ \\
\hline 1-Head ache & 9.18 \\
\hline 2- Fever & - \\
\hline 3-Rhinitis & 10.20 \\
\hline 4-Other & 1.02 \\
\hline 5-No effect & 82.65 \\
\hline
\end{tabular}

\section{Discussion}

In present cross sectional study there were maximum female $(75.5 \%)$ participants who were married and employed. This finding again supports the need to look young and smart at work field. Total 98 subjects with mean age of $28.04 \pm 4.01$ years were habitual hair dye users irrespective of gender. It is supportive to similar type of study where the mean age at which both genders started hair dyeing was 27 years, while it was 16 years in a study conducted on Danish adult population and 40-49 years in Korean population. $(6,7,8)$ The youngest volunteer using hair dye in our study was 20 years. However, in a study done in Riyadh, the practice began at an age as young as 15 years.(9)

Purpose to colour hair for youthful appearance was noted in $76.53 \%$ of population. The desire to look younger is widespread which is being reinforced everyday by the media and the social media. This is supported by survey response study done by Mrinal G. at Jammu and Kashmir, India (6). Only fashion or cosmetic purpose was observed in $56.12 \%$ population where as $21.43 \%$ genuinely felt need of dyeing hair due to greying. It is directly suggestive of aesthetic value of hair colour irrespective of age.

Maximum numbers of subjects $(n=57)$ were colouring hair with a gap of more than one month. While the pattern or area covered during dyeing was global in 55 participants. This pattern is suggestive of colouring hair after a longer period. A few dyed hair at an interval of 7-15 days where need was root touch up.

Though in our survey $90.82 \%$ perceived that hair dyeing itself was unsafe and hair dyeing products can cause various adverse effects, including allergic contact dermatitis, hair colouring was done by them( $(\underline{10})$. Also $80.61 \%$ population knew Natural / Herbal dyes are safe, only36.74\% used herbal dye whereas $63.27 \%$ used synthetic dye. This can be due to user friendly application and long lasting effect of synthetic dyes. To cater the need of increased demand of the market for natural, safe, efficient and long lasting hair dye; Ayurveda science can provide the best solution.

The need for repeated dyeing (75\%) because of cosmetic value and gray hair with observed level of satisfaction $(81 \%)$ after dyeing hair was similar to previous study (11).

Adverse effects during dyeing at scalp as itching and burning only up to $17.35 \%$ \& $12.25 \%$ respectively. No Side effects during dyeing at skin in 89 $\%$ population with minor redness in few subjects. This may be attributed to awareness and conscious selection of suitable and tested hair dye by that individual. No harmful effects were noticed on Eyes other than redness and burning upto $12 \%$ participants. Rhinitis and Headache were the two generalised physical symptoms in dye users (up to $10 \%$ ). Despite such experiences all 98 participants continued hair dyeing.

A side effect of dye on body of hair like dryness, hair fall, and change of texture was the important finding of this study. This finding indicates 
Mrudula Vinayak Joshi et.al., Knowledge, practice and adverse reactions amongst Hair dye users - A cross sectional study

the need of future study with respect to composition of hair and type of hair dye. It is also required to create awareness in children and younger generation to avoid undue, excess use of hair dye.

\section{Conclusion}

Overall $60 \%$ of the population was found as regular dye users in PCMC region. Majority population started hair dyeing at very early age using Chemical dyes even though they perceived herbal dyes were safe. They continued hair colouring despite adverse reactions, due to increasing life-achievements, the desire to look youthful. This precipitates aesthetic and remedial importance of hair colour and need of research in developing safe, natural hair dye for this ever increasing condition of PHG.

Global scenario is now shifting towards the use of safer, nontoxic natural product with traditional use, attempts are required to develop herbal hair dye devoid of any chemical, containing few traditionally used herbs and modifiers. It is seen that powder hair dyes with herbal ingredients are prominently in use in south east Asia and it is getting acceptance in other developed markets like EU and Americas (12).

As a holistic science, Ayurveda can provide the effective solution to this question without adverse effects and also preventing the recurrence.

\section{References}

1. Phadatare Suvarna P et al. / Comparative Study of Dyeing Efficiency and Retention Capacity Of Herbal Hair Dyes, Int. J. Res. Ayurveda Pharm. Mar - Apr 2013. 4(2), 198-202

2. Zapolanski T, Jacob SE. Para-Phenylene diamine. Dermatitis 2008; 19:20-21.

3. Yadavji Trikamji Acharya, Agnivesha's Charaka Samhita with Ayurved Deepika commentary of Chakrapanidatta, Ed., Chaukhambha Sanskrit Sansthan, Varanasi, Reprint, Sutrasthana,2014; 5/99.

4. Hari Sadasiva Sastri Paradkar, Vagbhata's Ashtanga Hridaya Samhita with the commentaries of Sarvangasundara of Arunadatta \& Ayurveda
Rasayana of Hemadri; Ed., Chaukhambha Sanskrit Sansthan, Varanasi; Reprint; 2012;Uttarsthan 23/29

5. Tripathi B, Vagbhata's Ashtanga Hridaya Samhita edited with Nirmala hindi commentary, Chaukhamba Sanskrit Pratishthan; Oriental Publisher and distributors, Delhi; 2007, 24: 1056-1064.

6. Gupta M. A study of knowledge, attitude and practices regarding hair dye use among general population. Our Dermatol Online. 2017; 9 (1):15-18. DOI: $10.7241 /$ ourd.20181.4

7. Sosted H, Hesse U, Menné T, Andersen KE, Johansen JD. Contact dermatitis to hair dyes in a Danish adult population: An interview-based study. Br. J. Dermatol. 2005;153:1325. [PubMed] [Google Scholar]

8. Kim JE, Jung HD, Kang H. A survey of the awareness, knowledge and behavior of hair dye use in a Korean population with gray hair. Ann Dermatol. 2012;24: 274-279. [PMC free article] [PubMed] [Google Scholar]

9. AlGhamdi KM, Moussa NA. Knowledge and practices of, and attitudes towards, the use of hair dyes among females visiting a teaching hospital in Riyadh, Saudi Arabia.Ann Saudi Med. 2011; 31 : 613-619. [PMC free article] [PubMed] [Google Scholar]

10. Chey WY, Kim KL, Yoo TY, Lee AY. Allergic contact dermatitis from hair dye and development of lichen simplex chronicus. Contact Dermatitis. 2004;51:58. [PubMed] [Google Scholar]

11. Patel D, Narayana S, Krishnaswamy B. Trends in use of hair dye: a cross-sectional study. Int $\mathrm{J}$ Trichology. 2013 Jul;5(3):140-3. doi: 10.4103/0974-7753.125610. PMID: 24574693; PMCID: PMC3927172.

12. Jayaganesh Sankar, Sudhir Sawarkar, Jainendra Malakar, Bachan Singh Rawat and Mohammed Asif Ali, Mechanism of Hair Dyeing and Their Safety Aspects: A Review. Asian Journal of Applied Sciences, 2017. 10: 190-196. DOI: 10.3923/ ajaps..190.196. URL: https://scialert.net/abstract/? doi=ajaps.2017.190.196. 